\title{
Decoding the English standard operating procedures for dentists and the dental industry
}

\author{
Edmund Proffitt ${ }^{1}$
}

\section{Key points}

Standard operating procedures for dental practices to undertake a phased transition towards the resumption of a full range of dental provision in England have now been published by the NHS/Office of Chief Dental Officer England.
The document essentially fulfils the role of a 'resumption blueprint' for dentistry in England and could also be seen as a catalyst for change in how dentistry is delivered going forwards through the COVID-19 pandemic and beyond.
Resumption of treatment will be risk-assessed throughout and will bring a number of very significant changes in the way in which dentistry will be delivered, with a heavy emphasis on personal protective equipment and strict infection prevention and control.

\begin{abstract}
The recently published Standard operating procedure: transition to recovery for the resumption of dental treatment in England may prove to be not only the blueprint for the resumption of face-to-face treatments, but also a possible catalyst for change. Seemingly, the raison d'être of the new standard operating procedures is not just to outline the detailed procedures for kick-starting dentistry, but also to support practices through transition and the shift towards a preventative and minimally invasive clinical approach that meets the current clinical challenges, and possibly then goes beyond. Detailed guidance is also provided throughout the document, including the provision and type of required personal protective equipment and clinical guidelines.
\end{abstract}

Alongside dental professionals, the dental industry welcomed the Chief Dental Officer (CDO) England's 'Resumption of Dental Services in England' letter dated 28 May 2020, ${ }^{1}$ asking that all dental practices commenced opening for face-to-face care from Monday, 8 June 2020. It highlighted that resumption would be dependent on practices having assessed that they have the necessary personal protective equipment (PPE) and infection prevention and control (IPC). Initially, this 'English approach' surprised many who were expecting a more phased strategy as previously outlined across the devolved administrations.

Backing up the 'return' letter was the seemingly protracted publication of Standard operating procedure: transition to recovery on 4 June 2020. ${ }^{2}$ This 61-page document essentially fulfils the role of the key 'resumption blueprint'.

${ }^{1}$ Chief Executive, British Dental Industry Association, Mineral Lane, Chesham, Buckinghamshire, HP5 1NL, UK. Correspondence to: Edmund Proffitt

Email address: EdmundProffitt@bdia.org.uk

Accepted 15 June 2020

https://doi.org/10.1038/s41415-020-1853-7
It may, however, prove to be not only the blueprint for the resumption of face-to-face treatments, but also a catalyst for change as CDO England, Sara Hurley, outlined in her introduction to the document: 'The limitations in AGPs present an opportunity to re-think our approach to care pathways. The patient-focused, team-delivered minimum intervention oral healthcare philosophy helps in taking on the current challenges in delivering dental care. The philosophy with its four interlinking domains of identifying the problem, prevention \& control, minimally invasive treatments and suitable recall strategies dependent upon longitudinal disease susceptibility, underpins all disciplines of dentistry. Whilst dental teams may use a variety of acceptable techniques to risk manage care, the guidelines for remote consultations, non-AGP periodontal treatment, restorative and paediatric dental care contained in this SOP provide an aide memoire to best practice, minimising AGPs and delivering quality health outcomes.' $^{2}$

Seemingly, the raison dêtre of the new SOPs is not just to outline the principles and procedures for kick-starting dentistry, but also to support practices through transition and the shift towards a preventative and minimally invasive clinical approach that meets the current clinical challenges, and then goes beyond. It could be seen as a vehicle to herald what the CDO England has described as a 'COVID-19 legacy hallmarked by a determined revision of the current activitydriven clinical approach, optimising time with patients and delivered as an integrated oral health team. ${ }^{3}$ Beyond the scope of the SOP document, there also appears to be an appetite for contract reform to support this 'revision' going forwards.

The document anticipates and paints a picture of a phased approach to full resumption based on risk management and outlines the steps in some detail, with those steps thoroughly dependent upon risk assessment and the availability of PPE at all stages. From an industry perspective, the British Dental Industry Association (BDIA), supported by its members, has worked hand in hand with the Office of the CDO (OCDO) England, the BDA and many other professional organisations, government agencies and representative parties every step of the way towards resumption, and 
has advised on PPE and product availability regularly.

In the short term, PPE provision remains a challenge for the dental (and broader medical) devices supply chain, both in terms of availability and cost. However, things do seem to be easing gently as time moves on, but we cannot completely eliminate the possibility of some further future supply chain 'wobbles', and should anticipate and prepare for contingencies as the profession and industry move forwards together along the road to resumption. We must not, of course, disregard the possible impact of the end of the Brexit 'transition period' as the UK currently remains set to leave the EU on 31 December 2020. However, perhaps we can take heart in some words from the CDO England, who wrote: 'I strongly argue that dental care has not run out of road and that COVID-19 presents the opportunity to shift gear and change lanes. ${ }^{3}$

Moving forwards, with an initial helping hand of PPE from the Department of Health and Social Care, the industry is confident that dentistry's highly professional supply chain can fully support the accelerating pace of resumption. However, at this point in time, both the profession and industry cannot predict what the rate of recovery and treatment volumes will be, or whether there may be additional lockdowns or cessations of treatment based on future disease patterns. With the need for additional PPE and aerosol generating procedure (AGP) mitigation activities, patient throughput remains a challenge. This will inevitably have implications for income generation and the sustainability of both dental practices and the dental industry. Anecdotal estimates of around $30-40 \%$ of historic patient activity resuming over the coming months will mean that practices and the industry will face some significant economic challenges along the 'road to resumption'.

Moving back to PPE, the SOPs do contain much technical detail of interest and relevance to all of us. Its protocols remain fully in line with government advice and Public Health England (PHE) remains the final word on PPE. The SOPs contain no real surprises in this area but are helpful in terms of detailing requirements. Incidentally, the document lists what constitutes both AGP and non-AGP activities in its 'Section 3'.

For non-AGP care, standard infection control precaution PPE consisting of eye protection, disposable fluid-resistant (type IIR) surgical masks, disposable aprons and gloves are cited.

For all AGPs, to prevent aerosol transmission, disposable fluid-repellent gowns (or approved equivalent), gloves, eye/face protection and an FFP3 respirator should be worn by those undertaking or assisting in the procedure. However, it is important to note that the SOPs do allow for a fit-tested FFP2 respirator to be used where FFP3s are not available. In the real world of current global PPE supply, this means that the much more readily available FFP2 respirators will be the backbone of the resumption of any AGP-based activity going forwards.

Subsequent to the publication of Standard operating procedure: transition to recovery, on 9 June 2020, NHS England issued updated guidance for healthcare providers, including dental practices, aimed at minimising nosocomial infections in the NHS. ${ }^{4}$ The guidance states that, from 15 June 2020, all staff in dental practices will be required to wear a surgical face mask when not in PPE or in a part of the facility that is COVID-secure. PHE has also responded to requests from the industry and has provided the BDIA with a detailed interpretation of COVID-19 guidance for dental industry maintenance, and service engineers and technical staff attending dental practices, so that surgeries can rest safe in the knowledge that the industry has its own bespoke PHE guidance on appropriate PPE to wear when visiting practices.

The SOP document also clarifies sessional use of PPE, stating that fluid-resistant (type IIR) surgical masks and eye protection can be used for a session of work rather than a single patient or resident contact. It also confirms that FFP2/N95 respirators are suitable for sessional use in dental practice, adding that a full-face visor changed between patients will protect the respirator from droplet/ splatter contamination. The procedures also state that, although good practice, there is no evidence to show that discarding disposable respirators, face masks or eye protection inbetween each patient reduces the risk of infection transmission to the health worker or the patient.

Among the procedures, guidance, illustrations, tables and appendices featured, there are, of course, some central and fundamental tenants and core guidance which are becoming part of the COVID-19 era's dental mantra. Where dental treatment is planned, care planning should focus on achieving stabilisation, intervention should be kept to a minimum to reduce exposure risk, and AGPs should be avoided where possible and only undertaken if the dental service has the appropriate PPE. Treatment should be completed in the minimum number of visits possible, and when an AGP has been undertaken, it is recommended that the room is left vacant for one hour for a neutral pressure room before cleaning is carried out. This period did raise a number of eyebrows and there are differing times cited in guidance from other countries. ${ }^{5}$ It is understood that additional mitigation means and technologies could play a part in reducing this time, subject to suitable risk assessment.

Of course, patient flow and practice layout should be considered in order to comply with social distancing measures throughout the practice, with reception use minimised, digital appointment booking (online, e-mail) used, the consideration of fitting physical barriers at reception (for example, Perspex shields) and arrangements for contactless card payment where possible. The dental industry will endeavour to render all possible help and support to practices through all of these steps.

Additional training of staff may be necessary and should be provided before recommencing any dental provision. Areas highlighted include rubber dam placement, four-handed techniques, decontamination and IPC, remote consultation and triaging, training in new IT software tools (for example, online medical history software), and scenario-based team training of new policies and procedures. Again, the dental industry will endeavour to render all possible help and support to practices through all of these steps and processes.

As services resume and practice capacity to provide care hopefully accelerates and increases, there will be a demand for a broader range of clinical activities and thus support from the industry. Areas of support to the profession will include tools to assist in preventative and self-care measures, delivered in line with Delivering better oral health ${ }^{6}$ and in AGP mitigation. The SOP document highlights and addresses areas of AGP mitigation, including the use of hand instrumentation/ scaling and non-AGP periodontal treatment, simple dental extractions, caries excavation with hand instruments, caries removal with slow-speed and high-volume suction, the placement of restorative material, orthodontic treatments and paediatric oral health, 
including stainless steel crowns and diamine fluoride applications. Detailed appendices provide guidance in these areas.

By working through the SOPs, the dental industry can identify just how and where it can support the resumption of more widespread dental treatment in the community and, importantly, explore new opportunities and areas of support for practices and the profession going forwards, as dentistry takes this opportunity to re-think and re-evaluate its approach to care pathways.

Resumption is a partnership between many groups: the patient, the dental team, the dental industry, the NHS, the BDA and other professional bodies and organisations, regulators and the government (apologies to any others that I have missed out). While there are a plethora of ideas and views across these groups, the most important thing is that they share common goals.

\section{References}

1. Chief Dental Officer England. Resumption of Dental Services in England. 2020. Available at https://www. england.nhs.uk/coronavirus/wp-content/uploads/ sites/52/2020/03/Urgent-dental-care-letter-28-May. pdf (accessed June 2020).

2. Office of Chief Dental Officer England. Standard operating procedure: transition to recovery. 2020. Available at https://www.england.nhs.uk/coronavirus/wp-content/ uploads/sites/52/2020/06/C0575-dental-transition-torecovery-SOP-4June.pdf (accessed June 2020).
3. Hurley $S$. Why re-invent the wheel you've out of road? BrDent J 2020; 228: 755-756.

4. NHS England and NHS Improvement. Minimising nosocomial infections in the NHS. 2020. Available at https://www.england.nhs.uk/coronavirus/wp-content/ uploads/sites/52/2020/06/C0586-minimisingnosocomial-infections-in-the-nhs.pdf (accessed June 2020).

5. COVID-19 Dental Services Evidence Review (CODER) Working Group. Recommendations for the re-opening of dental services: a rapid review of international sources - Version 1.1. 2020. Available online at https:// oralhealth.cochrane.org/news/recommendations-reopening-dental-services-rapid-review-internationalsources (accessed May 2020).

6. Public Health England and Department of Health. Delivering better oral health: an evidence-based toolkit for prevention. 2017. Available at https://assets. publishing.service.gov.uk/government/uploads/ system/uploads/attachment data/file/605266/ Delivering_better_oral_health.pdf (accessed June 2020) 